\title{
Origen de la ventaja competitiva y estrategia competitiva hacia una formulación dinámica
}

\section{Origin of competitive advantage and competitive strategy towards a dynamic formulation}

\author{
Saulo Edison Murillo Cornejo (*)
}

\begin{abstract}
RESUMEN
En este artículo se tratan aproximaciones teóricas acerca de las fuerzas competitivas, ventana competitiva y la estrategia competitiva y se responde, mediante el análisis de casos de empresas, preguntas frecuentes para académicos y empresarios como ¿Cuál es el origen de la ventaja competitiva y la estrategia competitiva? ¿El porqué de la formulación dinámica de la estrategia competitiva? Finalmente se define la formulación dinámica de la estrategia competitiva.
\end{abstract}

Palabras Clave: Fuerzas competitivas, Ventaja Competitiva, Estrategia competitiva.

\section{ABSTRACT}

This paper discusses theoretical approaches about the competitive forces, competitive advantage and competitive strategy and respond by analyzing the cases of companies, frequently asked questions for scholars and entrepreneurs as What is the source of competitive advantage and strategy Competitive? The reason for the dynamic formulation of competitive strategy. Finally define the dynamic formulation of competitive strategy.

Key words: Competitive Forces, Competitive Advantage, Competitive Strategy.

\section{INTRODUCCIÓN}

\section{ANÁLISIS COMPETITIVO}

El análisis de fuerzas competitivas se basa en las cinco fuerzas competitivas [1] y el análisis de red de valor [2][3]. Dentro del análisis del entorno, se perciben los factores: políticos, tecnológicos, económicos, sociales; además, se encontrarán interactuando los agentes competitivos: Clientes, competidores, complementadores, proveedores, Gobierno, Organizaciones no económicas. El análisis parte del comportamiento de los Agentes competitivos ante la influencia de los factores de entorno (figura 1).

El marco de las fuerzas competitivas orienta a identificar el resultante de la combinación de agentes y factores: políticos, tecnológicos, económicos, sociales; que antes fueron definidos. En ese sentido, el análisis competitivo exige a las empresas evaluar parámetros de negociación con los agentes competitivos. Las decisiones estratégicas de los agentes se orientan a la consecución de beneficios en las diferentes transacciones y acuerdos; es por ello que, un equilibrio ganar-ganar, donde las posiciones estratégicas adoptadas no deriven en arrepentimientos futuros y la composición sistémica conduzca a una sinergia con mayor valor aportado.

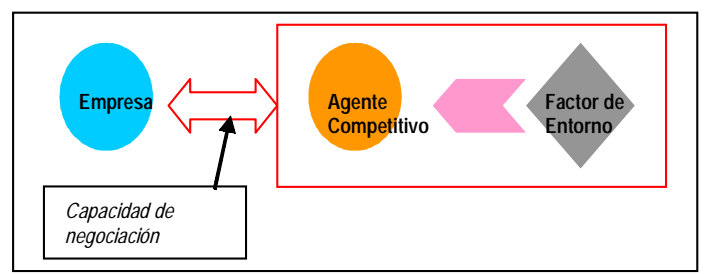

Figura 1

\footnotetext{
(*) Estudiante de Ingeniería de Sistemas y Computación. Facultad de Ingeniería. Universidad Católica Santo Toribio de Mogrovejo
} 


\section{EMPRENDIMIENTO EN LA INDUSTRIA}

La frontera de productividad, se puede definir como el estado dinámico no alcanzable, consiste en el estado de prácticas idóneas, que incluya la totalidad de las mejores prácticas existentes en un momento dado [4]. También define la eficacia operativa que consiste en realizar actividades similares mejor que los rivales; la operativa comprende la eficiencia, además se refiere a las prácticas que permiten utilizar mejor los recursos. Además, en su teoría sostiene que la mejora constante de la eficacia operativa es necesaria para conseguir mejor rentabilidad. Es un estado no alcanzable, un estado idóneo puesto que la frontera de la productividad se expande constantemente a medida que se desarrollan nuevas tecnologías y enfoques gerenciales y a medida que se dispone de nuevos insumos. Al avanzar hacia la frontera, es frecuente que las empresas mejoran simultáneamente. Cuando la empresa mejora su eficacia operativa se acerca a la frontera. La frontera se traslada hacia el exterior por la presencia de tecnología, nuevos sistemas de gestión y nuevos medios.

En cierto punto del tiempo de la evolución de la industria, muchas empresas compiten imitándose unas a otras, donde conseguirá una mayor posición aquellas empresas que reaccionen primero al entorno competitivo. Pero resulta que para otras empresas lograr en primero lugar eficacia operativa tratando ganar un margen de utilidades aceptable le implicará esfuerzo no recompensado dado que ya existen empresas posicionadas en el mercado. En este punto se produce lo que porter denomina una competencia destructiva; también para ciertos sectores los denominados Océanos Rojos [5]. La finalidad de la empresa es competir contra otros alcanzando poco a poco la cuota de mercado conforme aparecen nuevos competidores, las posibilidades de beneficio y crecimiento disminuyen, los productos se estandarizan al máximo y la competencia se vuelve destructiva. Los rivales pueden copiar rápidamente cualquier nueva posición de mercado y la ventaja competitiva, es el mejor de los casos, temporal [4][5][6]. Esto último sucede cuando las empresas diseñan sus estrategias enfocando el posicionamiento y la competencia.
Porter, M., afirma que entre las empresas existen diferencias de eficacia operativa. Estas diferencias son una fuente importante de diferencias de rentabilidad entre competidores, porque afectan directamente a su posición relativa de costos y a su nivel de diferenciación. La competencia por mejorar la eficacia operativa hace que la frontera la eficacia operativa hace que la frontera de productividad se desplace hacia el exterior y se eleve el listón para todos. Aunque esta competencia trae una mejora absoluta de la eficacia operativa, nadie aventaja a nadie respecto a una posición estratégica. A medida que los rivales se imitan unos a otros las estrategias van convergiendo, y la competición se convierte en una serie de carreras por vías idénticas en las que nadie puede ganar. La competencia basada exclusivamente en la eficacia operativa es mutuamente destructiva, y conduce a una guerra de desgaste que únicamente puede detenerse limitando a la competencia.

Zook, C. en [7] afirma que muchas empresas cambian su Core Busniess, aquella definición de negocio; durante su proceso de crecimiento muchas ven que el Core Business actual no produce los rendimientos esperados y optan por nuevos negocios, pues observan que su core ha perdido relevancia en el mercado, descubren nuevos Core Busines. El estudio de Zook, C. apuesta por el cambio empresarial, por una reinvención. Hamel, G. y Parlad, C.K. en [8], también afirman que muchas empresas deben reinventarse, deben aprovechar las oportunidades del entorno, enfocándose y participando de ellas, las cuales llevan a la reinvención.

Oster, S., en [6] afirma que la actividad emprendedora es una forma de que una empresa obtenga rendimientos más elevados que sus competidoras colocadas de forma similar, es ser la primera y aprovechar las nuevas oportunidades lucrativas. Por ejemplo en los océanos azules, en [5], existen posibles mercados, para una nueva gama de productos y servicios. En la evolución de la industria, estos productos, industrias o mercados, siguen el mismo proceso. Las empresas deben posicionarse y cuidar su posición, lograr lo que se denomina una posición sostenible. Las empresas tratarán de evitar que nuevas empresas le aventajen con estrategias, probablemente estrategias 
genéricas de negocio (costo, diferenciación, enfoque) o de gobierno (alianzas, cooperaciones, fusiones), tratando en lo posible obtener una posición sustentable difícil de alcanzar o imitar.

\section{ORIGEN DE LA VENTAJA COMPETITIVA}

Porter, M, en [9], define la capacidad como la coordinación de actividades, donde en la definición de organizaciones basadas en procesos, las actividades constituirían un conjunto de procesos los cuales consumen recursos. Amit, P. y Schoemaker, R. en [10], mencionan que ciertas capacidades están contenidas dentro de activos estratégicos. Los activos estratégicos, son un conjunto de recursos y capacidades asociadas, las cuales en el mercado son difíciles de imitar; así, a partir de este conjunto de recursos y capacidades la empresa consigue su ventaja competitiva.

Hamel, G. y Prahalad, C.K., en [11], proponen la creación de una cartera de competencias, y que estás proporcionen valor a los productos o servicios de las unidades de negocios. Las empresas deben desarrollar sus Core Competences o competencias medulares, que consisten en conjunto de prácticas, habilidades y tecnología complementarias que permiten a una empresa genera valor.

En el complejo de interacciones en el mercado, la empresa desarrolla una plataforma de activos estratégicos que refuerzan la ventaja competitiva. La ventaja competitiva basa su unicidad en los atributos emergentes de su estructura sistémica de una serie de componentes interrelacionados que forman parte de la ventaja competitiva: las negociaciones con los agentes, la cartera de competencias medulares, y las actividades singulares (figura 2).

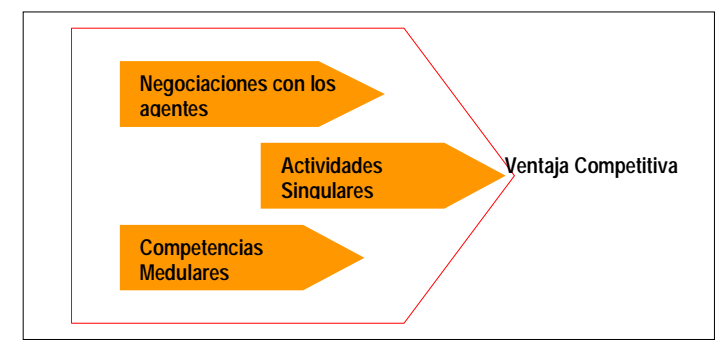

Figura 2
Las negociaciones con los agentes es igual de productiva para la gestión de la ventaja competitiva. Un caso de negociaciones, competencias medulares y actividades singulares es el caso de Sun Microsystems e IBM, quienes unen sus carteras de competencias para un mejor desarrollo de productos y servicios; se podría decir lo mismo de Jboss de Red Hat, que se basa en los servicios y productos de Sun Microsystems. Lo anterior resulta en una fórmula que involucra proveedores, competidores y se vuelve relevante el tema de estandarización y de compatibilidad que antes se venía mencionando. Otro ejemplo notable es el de Oracle Corporation y Sun Microsystems; aunque actualmente Oracle ha adquirido Sun Microsystems, históricamente ambas empresas han venido trabajando de manera conjunta en diversos proyectos. Oracle amplia el uso de la tecnología Java.

\section{Sistema de negociaciones}

La combinación conforma un sistema de negociaciones diferenciada de sus competidores. La empresa involucra a los agentes económicos (figura 3) en sus actividades, los proveedores están relacionados con la gestión de aprovisionamiento. Por el lado de complementadores, los servicios y productos deben ser compatibles con los productos y servicios de otras empresas. Por el lado de los competidores, en una forma de trabajo conjunto es posible que eleven el atractivo de la industria y proporcionen mayor seguridad a los consumidores en caso de productos nuevos, siendo esto importante en un contexto de constante innovación. El papel del gobierno y de organización sin fines de lucro también

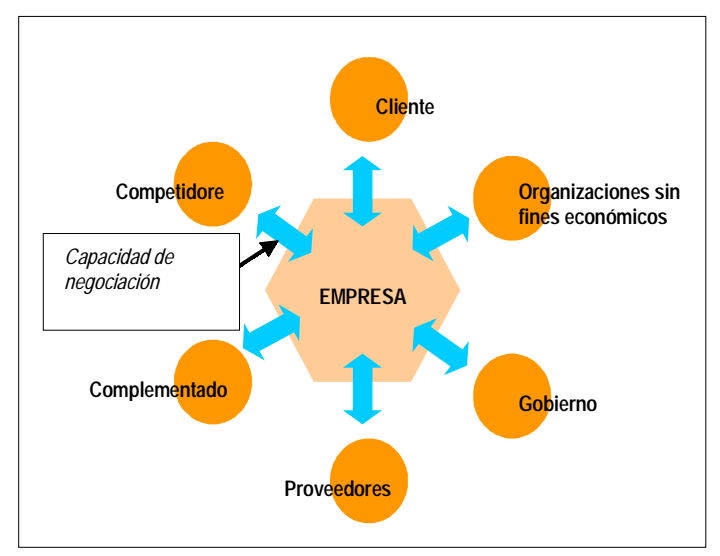

Figura 3 
juegan un papel importante tanto para llegar acuerdos como parea orientar tendencias, así lo demuestra la innovación de la computadora personal Unika Gyy, un producto ecológico diseñado dentro del contexto en los último años vividos acerca del cuidado del medio ambiente. Conviene observar las competencias de otras empresas tal como Unika Gyy desarrolla sus componentes ecológicos en China.

\section{Sistema de actividades singulares}

Es claro que según Porter, las empresas tienden a mejorar su eficacia operativa puesto que esto revela la mejora continua, y en consecuencia la reducción de costos, capacidad de diferenciación. Pero, no quiere decir que sea el camino hacia la mejor estrategia, dado que si bien alcanzan competitividad al acercarse a su frontera de productividad no están optando por acciones diferentes que las de sus competidores, ya que tienden a parecerse, si un negocio innovador.

Las empresas si bien tienden a buscar la eficacia operativa, pero para todas no es igual [4]. También se diferencian internamente. Las organizaciones todas difieren por haber experimentado el entorno de una forma distinta y la información que han obtenido la han internalizado de forma distinta. Diferencian internamente, no solo por la infraestructura sino por el desarrollo de su personal. Porter, M. resalta, menciona que las actividades deben ser singulares, muy diferentes a las de sus competidores. Estas actividades singulares, prestan una combinación de valor única. La propiedad emergente del sistema de actividades como característica distintiva de una empresa frente a sus competidores aumenta la probabilidad de mantener una poción sostenible, pues este sistema de actividades con valor único como resultante, constituye uno de los componentes de la ventaja competitiva.

\section{Competencias medulares}

Hamel, G. y Prahalad, C.K., en [11], definen competencias medulares como el producto del aprendizaje colectivo de la organización, especialmente las relativas al modo de coordinar las diversas técnicas de producción e integrar las múltiples corrientes de tecnologías.

Las competencias medulares presentan características que se deben rescatar y saber gestionar, el tiempo de desarrollo de una competencia, que implica el tiempo en que esta es consolidada es menor al tiempo en que el valor proporcionado subsiste y es un centro de grandes utilidades para la empresa en le mercado, no quiere decir que los tiempos coincidan exactamente. Por otro lado este tiempo de permanencia en el mercado es menor que el tiempo de vida que presenta la competencia medular. Sobre este último es claro que se debe discutir acerca de su tiempo de vida. Las competencias al resultar de la coordinación de habilidades y corrientes tecnológicas, siendo ambos componentes muy dinámicos, se debe notar que en algún momento se desarrollará una o nuevas competencias medulares que aportarán mucho valor a los centros de utilidades de la empresa y que sean motivo de la reinvención del negocio. Además, se debe también considerar que la competencia medular no siempre está dentro de la empresa, sino que puede estar fuera, localizada en otra empresa, tal vez una empresa proveedora, que eventualmente forme parte de la cartera de competencias y con su aporte resulte un mayor valor agregado por la empresa al mercado.

\section{ESTRATEGIA COMPETITIVA}

Para Oster, S. en [6], ésta comienza con el deseo de una organización de superar el desempeño del mercado. Consiste de un compromiso de emprender una serie de acciones en vez de otras. La ventaja competitiva es un conjunto de atributos, gestionados de forma eficaz, que apoyada por una estrategia competitiva logra para la empresa una posición sostenida. Para Porter, $M$. en [1], la estrategia se basa en un conjunto de acciones ofensivas y defensivas de la empresa para posicionarse, influir y anticiparse al mercado con el fin de crear y desarrollar una ventaja competitiva a largo plazo.

La estrategia como conjunto de acciones amplía el potencial de la ventaja competitiva, incluyendo las negociaciones con los agentes que desarrolle la empresa, la asignación de las 
competencias medulares a los centros de utilidades y la configuración de las actividades. Lo anterior se mide cuando son efectivas las negociaciones con competidores $y$ proveedores. Como el caso de Honda y General Motors en [12], cuando ésta última impulsa a sus proveedores a negociar una oferta con Honda, naturalmente en el marco de la innovación incrementar el atractivo de la industria para los clientes.

\section{FORMULACIÓN DINÁMICA DE LA ESTRATEGIA}

El problema de la planificación orienta a repensar cada cuánto tiempo se debe reinventar el negocio y la estrategia competitiva y qué acciones estratégica resultan ser más eficaces ante un mercado con agentes de aptitudes muy variantes. Reinventar e innovar el negocio e influir en la industria y por tanto contribuir a su reinvención, es para las empresas una de las vías o acciones estratégicas que impone un reto constante, este panorama puede significar una amenaza para ellas o una fuente de oportunidades. Tenemos el caso del Cirque du Soleil estudiado en [5], donde reinventaron el negocio en este caso no basándose en la competencia, no buscaron patrones repetitivos en la industria sino que reenfocaron el negocio siendo éste en último caso diferente de los demás, se basaron en las expectativas cambiantes del cliente.

En los casos de Dometic y Apple en [7], Dometic en principio solo orientó el negocio a las refrigeradoras siendo unas de sus ventajas su tecnología y la acogida que había obtenido; y posteriormente, para hacer más rentable el negocio, reorientó su negocio enfocándose en el cliente, vendiendo una gama de productos para la experiencia del cliente. Sus productos relacionados todos estaban orientados a las autocaravanas y no más a refrigeración por absorción.

El caso de Apple, es otro de los más ilustrativos y se puede apreciar, dentro del análisis de Zook, muchas de las afirmaciones acerca de la ventaja competitiva. Apple luego de haber estado compitiendo en el mercado de computadoras, reorientó el negocio hacia la industria de la música y reproductores digitales. Apple capitaliza tales fortalezas como gestión de marca, interfaz de usuario y software elegante y fácil de usar, tal como afirma Zook, que se ha convertido en su competencia para reorientar su negocio e innovar en la industria con productos y servicios como con Itunes Store. Acerca de su capacidad negociadora, se presenta también en el caso expuesto por Zook, su habilidad para la gestión de acuerdos con empresas discográficas y la gestión de derechos digitales. $Y$ definitivamente la prueba de la innovación y capacidad de aprendizaje se aprecia en la monitorización de las variaciones discontinuas de las expectativas de usuario con productos como Iphone y Apple TV.

En un contexto de constantes cambios la empresa necesita pilares para gestionar su ventaja competitiva. Sus habilidades más desarrolladas deben ser el la flexibilidad, el aprendizaje y la creatividad.

La flexibilidad se puede medir por la rapidez de comprensión de nuevos conceptos de puesta en marcha de nuevos actividades, pero también en la adaptación a nuevas formas de trabajar. Por otro lado, la creatividad exige alejarse de la rigidez mental, no moverse sobre lo sobre lo conocido apuntar a lo no considerado y adquirir nuevos puntos de vista [13]. La dirección juega un papel importante en la empresa al impulsar la flexibilidad para aumentar la productividad, se pueden citar ejemplos de grandes corporaciones, entre ellas General Motors, Motorota, Honda, General Electric y como el modelo japonés, que ya antes concebía la calidad como un requisito, en sus actividades tenía presente la flexibilidad [14].

La actividad emprendedora y la innovación tienden a reinventar los negocios y la industria. Un claro ejemplo se puede ver en el desarrollo de buscadores como Google, Excite, Lycos y Yahoo. Inicialmente los creadores de Yahoo que prácticamente ordenaron las páginas en directorios. La industria vendría definiéndose por los primeros buscadores y su fuente de ingresos era a través de la publicidad, Excite y Yahoo, entre otros buscadores como Altavista compitieron arduamente bajo una serie de servicios similares y olvidaron su cometido o finalidad enfocada en el usuario que era las búsquedas. El crecimiento de los usuarios estaba relación directa a los ingresos por publicidad así que de alguna manera los 
consumidores del servicio determinarían los ingresos de las empresas. A diferencia de estos buscadores, Google sabría como enfocar mejor el negocio centrado en el usuario en una interfaz de búsqueda y un eficaz y eficiente motor de búsqueda; con un sistema de negocio diferente, que aunque basado en la publicidad, no incomodaba con servicios, imágenes de publicidad a los usuarios. A los largo de su historia Google se ha preocupado tanto por los usuarios y sus clientes de quienes reciben sus ingresos con aplicaciones que llevan a una acuerdo de intereses. Los usuarios tiene un sistema de búsqueda cómodo y eficaz y el negocio de la publicidad no se afecta al contrario se incrementa. Posteriormente Google ha sostenido proyectos en función de su popularidad y ha sabido gestionar adquisiciones y desarrollo de aplicaciones enmarcadas en el desarrollo e innovación de tecnologías de información.

\section{Función de la estrategia}

En [15] los autores mencionan que la estrategia emergente está en relación con el aprendizaje organizacional, pues reconoce la capacidad de la organización para experimentar; en ese sentido, la estrategia emergente no obedece a un modelo predeterminado, sino que explica un patrón a medida que se van realizando ciertas acciones.

La estrategia no es una intención de acciones, no es un plan de acciones, tampoco se define como una serie consecuente de planes operativos, tácticas y eficacia operativa. La estrategia es una función de éxito, que tiene como vectores las intenciones orientadas al consumidor, de proporcionar valor al cliente, y la información de dominios interno (la empresa misma) y externo (el entorno competitivo). Dado que los tres parámetros son muy cambiantes, la estrategia no suele tener un orden proyectado, al menos no para el contexto actual. La estrategia persigue la mejor forma de explotar la ventaja competitiva, la cual se compone de negociaciones, actividades singulares y de competencia medulares, de manera que la gestión eficaz de la ventaja competitiva permitirá una diferenciación sostenible y productiva en el tiempo, se origina el valor de ella misma.

\section{CONCLUSIONES}

La formulación de la estrategia competitiva se relaciona directamente con el empleo de la ventaja competitiva; y los componentes de la ventaja competitiva interactúan necesariamente con son el dominio externo y el dominio interno.

La estrategia competitiva se orienta al consumidor (figura 4), ya que, se enfrenta a los cambios de sus expectativas en relación a la diversificación de producto o servicio y la relación calidad-precio; no obstante, no quiere decir que la estrategia no siga un orden de acciones y sea del todo emergente, sino que como un proyecto ordenado sigue periodos muy cortos. La estrategia competitiva, en ese sentido, no consiste de un orden de acciones deliberadas a largo plazo; en vez de ello innova con la misma frecuencia que el negocio deberá hacerlo.

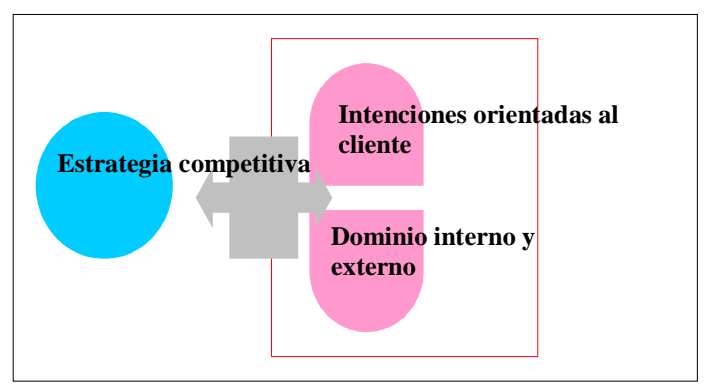

Figura 4

\section{REFERENCIAS BIBLIOGRÁFICAS}

[1] Porter, Michael (1985). Competitive Advantage, Free Press, New York.

[2] Brandenburger, Adam M. y Nalebuff, Barry (1995). Right Game: Use Game Theory to Shape Strategy. Harvard Business Review (Julio - Agosto):57-71.

[3] Brandenburger, Adam M. y Nalebuff, Barry (1996). Cooptencia. Grupo Editorial Norma. Bogotá, DC.

[4] Porter, Michael (1996). «What is Strategy», Harvard Business Review (Nov-Dec): 61-78.

[5] Kim, Chan y Mauborgne, Reneé (2005). Blue Ocean Strategy. Harvard Business School Press. Boston. MA.

[6] Oster, Sharon M. (2000). Análisis moderno de la competitividad. Oxford University Press. México. 
[7] Zook, Chris (2007) Descubra su próximo core business. Harvard Business Review (Abril): 86-97.

[8] Hamel, Gary y Prahalad, C. K. (1996 b). Competing for the future. Harvard Business School Press. Boston, MA.

[9] Porter, Michael (1991). «Towards a Dynamic Theory of Strategy», Strategic Management Journal,12: 95117.

[10] Amit, Raphael y Schoemaker, Paul J.H. (1993). Strategic assets and organizational rent. Strategic Management Journal, 14: 33-46.

[11] Hamel, Gary y Parlad, C. K. (1996 a). The Core Competence of the Corporation. Harvard Business Review (Mayo - Junio): 63-76.

[12] Peters, Tom (2006) Gestionar con imaginación. Ediciones Deusto. Barcelona.

[13] Sarasqueta, Antxón (2003). Una visión global de la globalización. EUNSA. Navarra.

[14] Ivancevich, John M.; Lorenzi, Peter y Skinner, Steven J. (1996) Gestión: Calidad y Competitividad. Mosby/ Doyma Libros. Santafé de Bogotá.

[15] Mintzberg, Henry; Ahlstrand, Bruce y Lampel, Joseph (1999) Safari a la estrategia: una visita guiada por la jungla del management estratégico. Granica. Buenos Aires.

E-mail: sauloedison_mc@hotmail.com 